\title{
Messsystem zur Bestimmung akustischer Kenngrößen stark absorbierender, transversal isotroper Kunststoffe
}

Rautenberg, Jens; Bause, Fabian; Henning, Bernd

Universität Paderborn, Institut für Elektrotechnik und Informationstechnik, Elektrische Messtechnik, Warburger Straße 100, 33098 Paderborn, Tel.: 05251 60-3018, FAX: 05251 60-3237

E-Mail: Rautenberg@emt.uni-paderborn.de

\section{Kurzfassung}

Es wird ein Wellenleiter basiertes Messsystem vorgestellt, mit dem anhand einer einzigen Transmissionsmessung alle Daten bestimmt werden können, die zur Simulation von Schallausbreitungsphänomenen in stark absorbierenden und schwach anisotropen Materialien erforderlich sind. Auf diese Weise ist es fortan möglich die akustischen Kenngrößen (winkel- und frequenzabhängige Schallgeschwindigkeiten und frequenzabhängige Absorption) verschieden konditionierter Materialproben (Temperatur, Feuchtigkeit, Alter) simultan zu erfassen.

Genutzt wird dazu die mehrmalige Modekonversion an den Mantelflächen eines hohlzylindrischen akustischen Wellenleiters, wonach ein Empfangssignal mehrere Signalgruppen aufweist, deren absolute Laufzeit, Laufzeitdifferenzen und Amplituden in Abhängigkeit der Materialkenngrößen stark variieren. Grundlegend für die realisierte und in diesem Beitrag beschriebene inverse Bestimmung der akustischen Kenngrößen ist ein ganzheitliches Modell der Schallanregung, -ausbreitung und -detektion, welches die schnelle Berechnung der Empfangssignale erlaubt. Die gezielte Variation der Modell-Eingangsgrößen führt schließlich zu einer bestmöglichen Übereinstimmung zwischen berechneten und gemessenen Empfangssignalen, sodass die final angesetzten Modell-Eingangsgrößen eine gute Approximation der gesuchten materialspezifischen akustischen Kenngrößen darstellen.

Im Rahmen dieses Beitrags werden der Messaufbau, wesentliche Teile der Modellierung sowie die grundsätzliche Vorgehensweise zur Anwendung der inversen Messmethode gezeigt. Anhand gemessener, berechneter und mittels Finiter Elemente Methode simulierter Signale wird das Verfahren für einen Werkstoff, spritzgegossenes Polypropylen, demonstriert.

\section{Einleitung und Motivation}

Akustische Messsysteme leisten oftmals einen hohen Beitrag zu Sicherung und Verbesserung industrieller Prozesse. Das Spektrum der Anwendung reicht dabei von der ultraschallbasierten Durchflussoder Konzentrationsmessung bis hin zur zerstörungsfreien Werkstoffprüfung bzw. Komponentenüberwachung. Die Komplexität der Prozesse erfordert stets problemangepasste bzw. optimierte Ultraschallsensoren, bei deren Design die Computersimulation einen wichtigen Beitrag leisten kann [1-3]. Für die realitätsnahe Simulation der Schallausbreitung in solchen Messsystemen ist die Verwendung realitätsnaher Materialkenngrößen bzw. -modelle und Simulationsparameter von grundlegender Bedeutung. Mit Hilfe der Longitudinal- und Transversalwellengeschwindigkeiten, Schallabsorption und Schallkennimpedanz lassen sich alle für die Simulation notwendigen Simulationsparameter elastischer isotroper Festkörper berechnen. Zumindest für die vielfach verwendeten Metalle und Keramiken genügt dabei die Annahme von Materialkonstanten sowie eines Rayleigh-Dämpfungsmodells, um die Schallausbreitung in einem eingeschränkten Frequenzbereich hinreichend gut zu simulieren [4].

Aus technischer sowie ökonomischer Sicht werden in akustischen Messsystemen aber vermehrt Kunststoffe eingesetzt. Sie bieten beispielsweise die Möglichkeit, konstruktive und akustisch funktionale Elemente (Anpasschichten, Vorlaufkörper, akustische Wellenleiter ...) in einem Körper zu verbinden. Diesem Vorteil stehen eine Reihe zu überwindender Schwierigkeiten gegenüber: So reichen die genannten akustischen Kenngrößen in der Regel nicht mehr aus, um das akustische Verhalten adäquat zu beschreiben. Ursache ist einerseits ein viskoelastisches Verhalten, welches zu frequenzabhängigen Kenngrößen führt. Andererseits können durch Spritzgießen glasfaserverstärkter Materialien anisotrope Werkstoffe entstehen, deren akustische Eigenschaften mit der betrachteten Raumrichtung variieren. Zudem schwanken Schallgeschwindigkeit, Dämpfung und Kennimpedanz, anders als bei Metallen, deutlich mit der Temperatur, dem Alter oder dem Wassergehalt. Die Bandbreite dieser Schwankungen gilt es im Vorfeld einer Entwicklung zu erfassen und bei der Dimensionierung eines ultraschallbasierten Sensors oder der Beurteilung eines polymeren Prüfkörpers zu berücksichtigen. 


\section{Bestehende und konkurrierende Messmethoden}

Angesichts der zu erwartenden starken Frequenzabhängigkeit der Materialkenngrößen und den typischerweise hohen Betriebsfrequenzen von Ultraschallsensoren scheiden statische und quasistatische Messmethoden (Zugversuch, Torsionsschwinger ...) zur Bestimmung der komplexen Kenngrößen aus. Es gibt aber eine Reihe verschiedener dynamischer Methoden zur Bestimmung der Steifigkeitstensoren, aus denen sich unmittelbar Schallgeschwindigkeit und Dämpfung ableiten lassen, wie später exemplarisch gezeigt wird. Eine Auswahl dieser Methoden soll nun vorgestellt werden, wobei zwischen EbeneWellen- und Geführte-Wellen-Ansätzen unterschieden wird.

Ebene-Wellen-Ansätze: Ein weit verbreiteter Ansatz zur Bestimmung der Elastizitätskonstanten plattenförmiger Probekörper ist die Immersionstechnik. Im Wasserbad wird dabei unter verschiedenen Winkeln die Transmission ebener Wellen durch den Körper untersucht. Anstelle einer Transmissionsmessung mit je einem Sende- und Empfangswandler ist auch ein Betrieb im Impulse-Echo-Modus mit nur einem Schallwandler möglich. Diese Back-Reflection-Technik wird im Vergleich zur Transmissionstechnik gerade für dicke Proben empfohlen [5]. Reddy et al. verwenden dabei als Modell die Christoffel-Matrix und schließen mit Hilfe der Kreuzkorrelationstechnik auf die Wellenlaufzeit bzw. Phasengeschwindigkeit. Diese ist schließlich das Kriterium zur inversen Modellidentifikation [5]. Die Methode wurde für isotrope und transversal isotrope Medien angewandt, wobei ausschließlich reelle Elastizitätskonstanten identifiziert wurden. Vishnuvardhan et al. bestimmten auf ähnliche Weise die E-Moduln eines orthotropen Composite-Materials [6]. Die Arbeitsgruppe konnte zeigen, dass die Methode nur eine geringe Sensitivität hinsichtlich der E-Moduln $\mathrm{C}_{12}$ und $\mathrm{C}_{44}$ (Voigtsche Notation) aufweist und diese somit nicht oder nur unzureichend genau zu bestimmen sind. Weitere Hinweise auf den nur „schwer experimentell bestimmbaren" und auch "großen Messunsicherheiten“ unterliegenden Modul $\mathrm{C}_{44}$ finden sich in den meist theoretischen Arbeiten von Theocaris [7]. Castaings et al. verwenden zwei separate Sende- und Empfangswandler und nutzen, ebenfalls an ebenen Platten, die Thomson-Haskell-Matrix-Methode, um den komplexen Transmissionskoeffizienten in Abhängigkeit der Frequenz und des Einfallwinkels zu beschreiben [8]. Es wurden isotrope und anisotrope Medien untersucht, wobei zumindest bezüglich der Realteile der E-Moduln eine geringe Messunsicherheit erreicht wurde. Ein leicht modifizierter Ansatz wurde von Sachse et al. unter dem Namen Point-Source/Point-Receiver Methode vorgestellt [9, 10]. Hier wird mittels fokussierter Laserstrahlung eine mechanische Welle angeregt und in Abhängigkeit des Winkels räumlich auf der Platte abgetastet. Durch die impulsförmige Anregung werden eine QuasiLongitudinal- und zwei Quasi-Transversalwellen angeregt, deren Eintreffzeitpunkte am Empfänger separiert werden müssen. Mit Hilfe eines inversen Ansatzes, basierend auf der Christoffel-Gleichung, wird dann das Materialmodell identifiziert. Probleme der Methode bestehen in der Identifikation bzw. Separation der verschiedenen Wellenarten und der Diskrepanz zwischen gemessener Gruppen- und für das Modell relevanter Phasengeschwindigkeit. Eine Laufzeitmessung spiegelt immer eine Gruppenlaufzeit wieder und kann streng genommen nur unter Freiraumbedingungen und Vernachlässigung von Dämpfungsphänomenen mit der Phasengeschwindigkeit als äquivalent angesehen werden. Letzteres Problem gilt auch für die Immersionstechnik.

Geführte-Wellen-Ansätze: In den letzten Jahren wurden vermehrt auch geführte Wellen eingesetzt, um die Elastizitätskonstanten eines Probekörpers zu bestimmen. Anders als bei den Ebene-Wellen-Ansätzen wird hierbei die räumlich begrenzte Ausdehnung der Proben konstruktiv zur Erhöhung der Empfindlichkeit genutzt. Vishnuvardhan et al. zeigen im Rahmen einer Sensitivitätsstudie zum Beispiel, dass die Geschwindigkeit der symmetrischen Grundmode in einem orthotropen Material kaum sensitiv, die antisymmetrische Grundmode dagegen deutlich auf den Modul $\mathrm{C}_{44}$ reagiert [11]. Um also auch diesen besonders kritischen Modul bestimmen zu können, ist es sinnvoll, einen multimodalen Ansatz zu wählen. In den meisten Ansätzen wird dementsprechend das dispersive Verhalten symmetrischer und antisymmetrischer Lamb-Wellen in plattenförmigen Probekörpern untersucht.

Im Wesentlichen unterscheiden sich die Geführte-Wellen-Ansätze darin, wie die dispersiven Kenngrößen des Wellenleiters bestimmt werden. Rogers nutzt beispielsweise einen Wedge-Transducer, welchen er entlang des Wellenleiters verschiebt, um durch Phasenvergleich mit dem Sendesignal die Randwellenlänge der Leaky-Lamb-Welle zu bestimmen [12]. Andere Forschergruppen nehmen an äquidistanten Punkten den zeitlichen Verlauf der Wellenbewegung am Wellenleiterrand auf und berechnen mittels $2 D$ Fourier-Transformation das gesuchte Dispersionsdiagramm der angeregten und am Wellenleiterrand sichtbaren Moden. Dabei wird aus der Zeitachse die Frequenzachse und aus der Raumachse die Achse der Wellenzahl [13]. Eine Forschergruppe um Sale et al. benutzt dagegen zur Reduzierung des technischen Aufwandes nur einen piezokeramischen Empfangswandler, um aus dem aufgezeichneten Zeit- 
signal auf Basis der Gabor-Wavelet-Transformation den frequenzabhängigen Verlauf der Gruppengeschwindigkeiten zu berechnen [14].

Als Wellenleitermodelle werden je nach geometrischer Form und Komplexität des Materialmodells analytische, halbanalytische oder numerische Modelle eingesetzt. Unabhängig von der gewählten Methode ist das Ziel die Beschreibung der Wellenausbreitung im Wellenleiter mittels sogenannter Dispersionsdiagramme, welche zum Beispiel die Beziehung zwischen räumlichem und zeitlichem Ausbreitungsverhalten (Wellenzahl und Frequenz) der ausbreitungsfähigen Eigenwellen (Moden) graphisch darstellen. Durch gezielte Variation der dem Modell zugrundeliegenden Eingangsparameter, welche idealerweise identisch mit den Materialkenngrößen sind, werden schließlich die berechneten mit den experimentell bestimmten Ergebnissen abgeglichen. Diese Vorgehensweise ist auch als inverse Methode bekannt.

Hybrider Ansatz: Die in diesem Beitrag verfolgte Vorgehensweise stell eine Kombination aus beiden gezeigten Ansätzen dar. In einem ersten Schritt werden aus einem geschlossen lösbaren Ebene-WellenAnsatz Startwerte für die sich anschließende inverse Methode auf Basis geführter Wellen bestimmt. Die sukzessive Vorgehensweise ermöglicht eine schnelle Konvergenz in dem schlecht gestellten numerischen Problem und stellt gleichsam sicher, dass bei der inversen Lösungssuche keine lokalen Optima angesteuert werden.

\section{Gleichzeitige Bestimmung der Longitudinal- und Transversalwellengeschwindigkeit}

Die grundlegende Idee zur gleichzeitigen Bestimmung der Longitudinal- und Transversalwellengeschwindigkeit auf Basis von Laufzeiten und Laufzeitdifferenzen stammt von Reynolds [15]. Das Verfahren wird in diesem Beitrag aufgegriffen, auf transversal isotrope Medien erweitert, und liefert damit die für die inverse Methode erforderlichen Startwerte. Mehr noch, es wird durch die geschlossene Lösung Bestandteil eines sehr effizienten Algorithmus zur Vorwärtsrechnung in jedem Iterationsschritt der inversen Methode. Angesichts seiner zentralen Bedeutung soll das Verfahren unter der Annahme isotroper Materialien kurz erläutert werden:

Reynolds Probekörper waren zylindrisch mit im Vergleich zur Wellenlänge großem Durchmesser. Gemessen wurde in Transmission von einer Stirnfläche des Zylinders zur anderen, wobei der mehrmalige Modenwandel im kreisrunden Stab genutzt werden soll (siehe Bild 1). So wird eine im Festkörper laufende ebene Longitudinalwelle $L$ beim Auftreffen auf eine Grenzfläche zur umgebenden Luft teilweise unter ihrem Einfallswinkel $\theta_{1}$ reflektiert, zum Teil aber auch konvertiert in eine Transversalwelle T', welche den Festkörper unter einem anderen Reflexionswinkel $\theta_{2}$ durchquert. Eine weitere in die Luft hineinge-

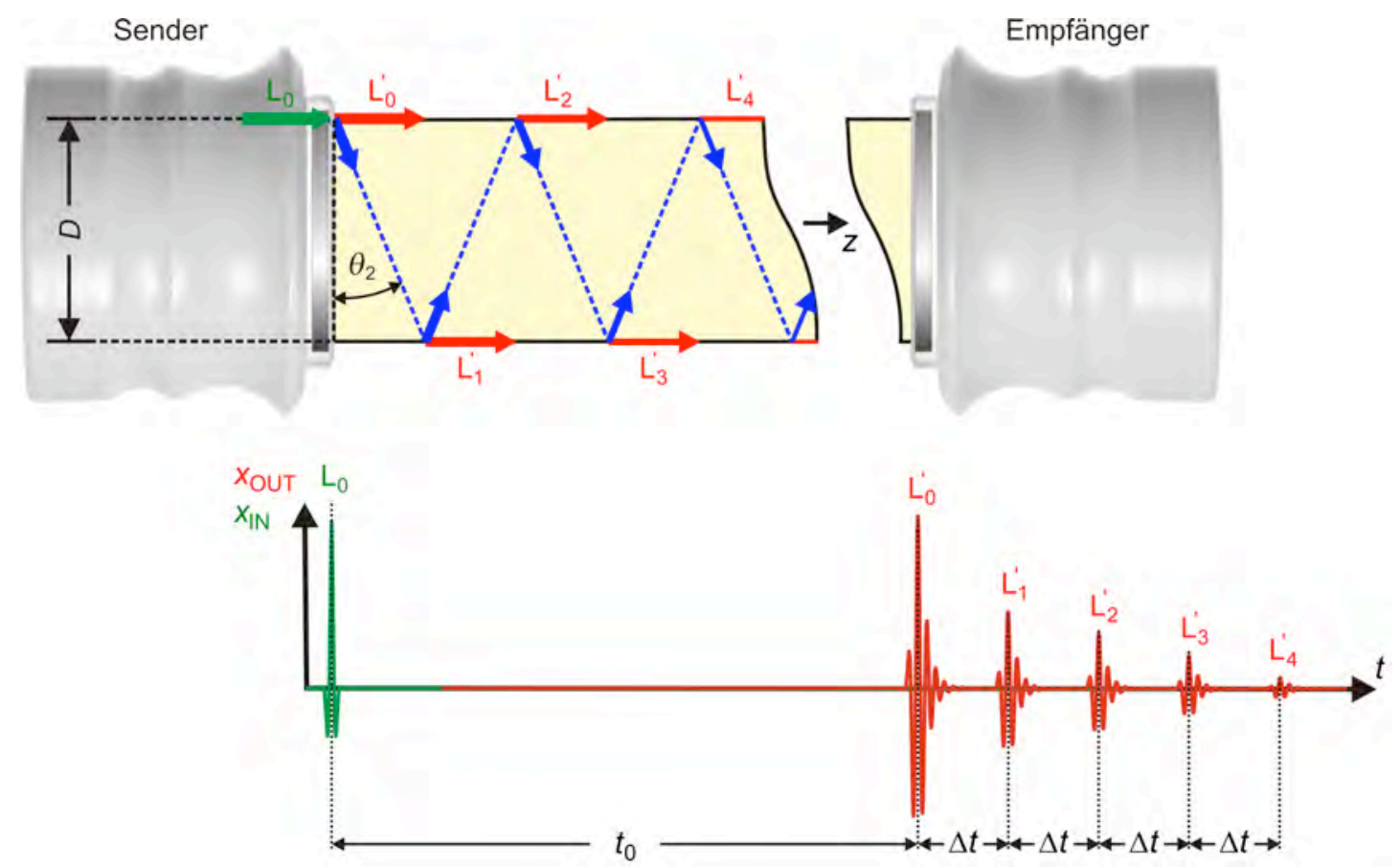

Bild 1: Versuchsaufbau nach Reynolds sowie schematische Darstellung der Wellenzahlvektoren $\mathrm{L}_{i}^{\prime}$ und $\mathrm{T}_{i}^{\prime}$ (oben) bzw. der Sende- und Empfangssignale (unten), wie sie sich nach den Modekonversionen ergeben können. 
brochene Welle kann angesichts des großen Unterschieds der akustischen Impedanzen vernachlässigt werden. Der Effekt ist auch umkehrbar, womit sich die Verhältnisse beim streifenden Einfall der initiierenden Welle $\left(\theta_{1}=90^{\circ}\right)$ nahezu wie in Bild 1 darstellen. Auf diese Weise kommt es am Empfänger zu einer Impulsfolge mit messbaren Laufzeiten $t_{0}$ und Laufzeitdifferenzen $\Delta t$. Mit diesen Signalkenngrößen ist es möglich, selbst bei Verwendung von ausschließlich Longitudinalwellenprüfköpfen sowohl die Longitudinalwellengeschwindigkeit $c_{\mathrm{T}}$ als auch die Transversalwellengeschwindigkeit $c_{\mathrm{T}}$ und zu bestimmen [15]. Mit $l$ und $D$ als Länge bzw. Durchmesser des Stabes gelten näherungsweise

$$
c_{\mathrm{L}} \approx \frac{l}{t_{0}}, \quad c_{\mathrm{T}} \approx c_{\mathrm{L}} \cdot\left(1+\left(\frac{c_{\mathrm{L}} \cdot \Delta t}{D}\right)^{2}\right)^{-0,5} .
$$

\section{Grenzen der Methode und Zieldefinition}

Bei Reynolds Herleitung von GI. 1 wurde die mehrmodige Schallausbreitung nicht herangezogen, obwohl dokumentiert ist, dass der Stab nicht zu dünn werden durfte, wenn man auswertbare Signale erhalten wollte (Cutoff-Frequenz). Man minimierte vielmehr den Fehlereinfluss durch die Wahl großer Durchmesser und schwach dispersiver Medien. Zwar gab es von Rayleigh, Pochhammer und Chree die theoretische Beschreibung und insbesondere von Mindlin Verfahren zur Approximation der Dispersionsdiagramme [16], angesichts mangelnder Rechentechnik konnte die mehrmodige Schallausbreitung aber keine Berücksichtigung finden. Diese Einschränkung gilt heute nicht mehr. Damit wird es möglich, auch stark materialdispersive und dämpfende Medien mit den dann notwendigerweise kleinen Probekörperdimensionen zu untersuchen.

Bild 2 zeigt ein Empfangssignal nach der Durchschallung eines Polypropylen-Zylinders (PP) im Vergleich zur Durchschallung von Polyetheretherketon (PEEK) bei nahezu gleicher Geometrie des Probekörpers und gleicher elektrischer Ansteuerung der Ultraschallwandler. Die Signale beginnen nahezu zeitgleich wohingegen sukzessiv folgende Signalgruppen verschiedene Laufzeiten und Amplituden aufweisen. Das zeitgleiche Eintreffen ist, wie in Gl. 1 gezeigt durch ähnliche Longitudinalwellengeschwindigkeiten bedingt, wohingegen die Variationen der Laufzeitdifferenzen auf unterschiedlichen Transversalwellengeschwindigkeiten schließen lassen. Ein weiterer offensichtlicher Un-

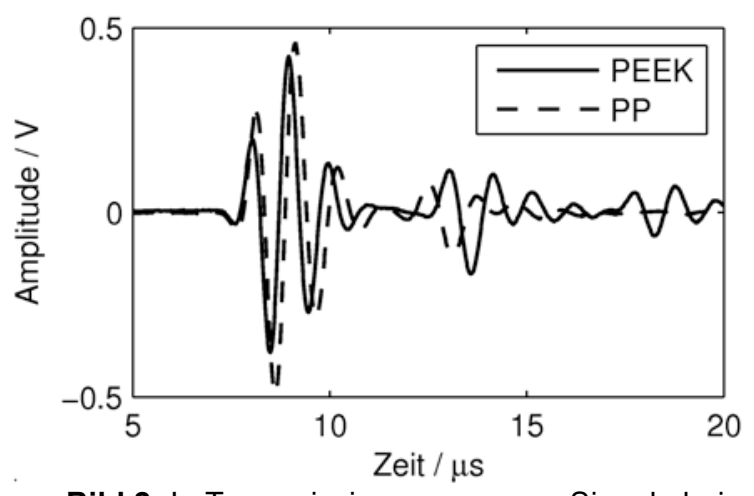

Bild 2: In Transmission gemessene Signale bei verschiedenen Materialien terschied ist beim PP die augenscheinliche Zunahme der Periodendauer bei gleichzeitiger Abnahme der Amplitude mit der Schalllaufzeit. Dies ist dann der Fall, wenn höherfrequente Anteile stärker gedämpft werden als niederfrequente. Die frequenzabhängige Dämpfung ist demnach beim PP deutlich stärker ausgeprägt als beim PEEK. Dieser Unterschied zeigt deutlich, wie wenig repräsentativ beispielsweise eine Nulldurchgangsdetektion für die Bestimmung der Laufzeiten bzw. Laufzeitdifferenzen in dispersiven Kanälen sein kann. Ein inverser Ansatz mit Vorwärtsrechnung der Signale kann dieses Problem auflösen. Zudem eröffnet sich die Möglichkeit zur Identifikation der materialspezifischen frequenzabhängigen Absorption. In diesem Beitrag soll weiterhin gezeigt werden, dass auf gleiche Weise auch transversal isotrope Medien auf ihre richtungsabhängigen Eigenschaften untersucht werden können.

\section{Akustische Kenngrößen}

Ausgangspunkt zur Berechnung der richtungs- und frequenzabhängigen Schallgeschwindigkeiten sowie der Schallabsorption ist die Annahme eines Materialmodells, beispielsweise linear viskoelastisches Verhalten in einer bestimmten Materialsymmetrie. Zentrales Element bei dieser Beschreibung ist der Steifigkeitstensor in Voigtscher Notation, mit dem alle möglichen Spannungen und Dehnungen eines infinitesimal kleinen Volumenfreischnitts über eine $6 \times 6$-Matrix in Beziehung gesetzt werden. Die einzelnen Elemente dieser Matrix sind die bereits oben verwendeten Moduln $C_{i j}$. In diesem Beitrag soll eine bestimmte Materialsymmetrie, die transversale Isotropie, vorausgesetzt werden. Der Steifigkeitstensor $\mathbf{C}$ stellt sich dann wie folgt dar [17]: 


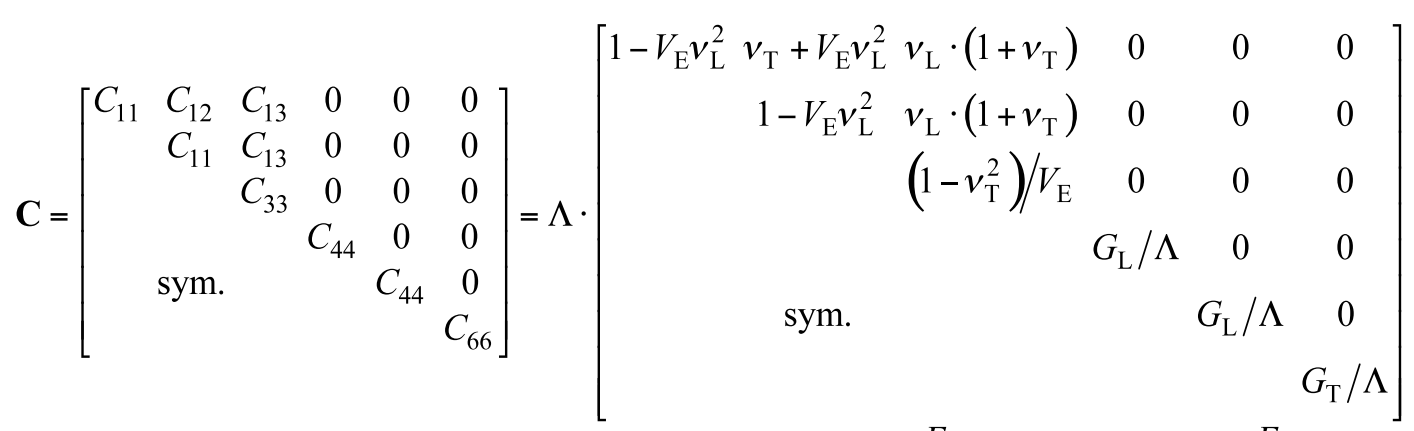

$$
\begin{aligned}
& \Lambda=\frac{E_{\mathrm{T}}}{\left(1+v_{\mathrm{T}}\right) \cdot\left(1-v_{\mathrm{T}}-2 V_{\mathrm{E}} v_{\mathrm{L}}^{2}\right)}, \quad V_{\mathrm{E}}=\frac{E_{\mathrm{T}}}{E_{\mathrm{L}}}
\end{aligned}
$$

Darin sind $E_{\mathrm{L}}$ und $E_{\mathrm{T}}$ die Elastizitätsmoduln in Längs- bzw. Querrichtung und $v_{\mathrm{L}, \mathrm{T}}$ die zugehörigen Querkontraktionszahlen. Das Besondere an der transversalen Isotropie ist, dass das Material zumindest hinsichtlich einer Drehung um die Längs-Richtung (hier 3-Richtung, im Modellkoordinatensystem meist die $z$-Achse) seine richtungsabhängigen Eigenschaften nicht ändert. Es gibt also eine isotrope $x y$-Ebene. Diese Anisotropie kann in vielen extrudierten Stangenmaterialien aber auch spritzgegossenen Körpern angenommen werden. Der Unterschied der mit "T" bzW. "L“ indizierten Koeffizienten wird dabei umso größer, je mehr oder längere Glas- bzw. Kohlefasern einem Kunststoff zugesetzt werden, vorausgesetzt es kommt zu einer guten und weitgehend homogen verteilten Vernetzung der beteiligten Materialien.

\section{Schallgeschwindigkeit (auch richtungsabhängig)}

Mit der Vorgabe eines Materialmodells lässt sich zum Beispiel die Christoffel-Gleichung aufstellen, deren Lösung unmittelbar zu den richtungsabhängigen Schallgeschwindigkeiten führt. Eine kompakte Darstellung findet sich beispielsweise in [18]:

$$
\begin{gathered}
c_{\mathrm{L}}^{2}(\varphi)=\frac{1}{2 \cdot \rho} \cdot\left[\left(C_{11}+C_{44}\right) \cdot \sin ^{2} \varphi+\left(C_{33}+C_{44}\right) \cdot \cos ^{2} \varphi+\Phi\left(C_{i j}, \varphi\right)\right] \\
c_{\mathrm{T}}^{2}(\varphi)=\frac{1}{2 \cdot \rho} \cdot\left[\left(C_{11}+C_{44}\right) \cdot \sin ^{2} \varphi+\left(C_{33}+C_{44}\right) \cdot \cos ^{2} \varphi-\Phi\left(C_{i j}, \varphi\right)\right] \\
\operatorname{mit} \Phi\left(C_{i j}, \varphi\right)=\left[\begin{array}{l}
\left(C_{11}-C_{44}\right)^{2} \sin ^{4} \varphi+\left(C_{33}-C_{44}\right)^{2} \cdot \cos ^{4} \varphi+\mathrm{K} \\
\mathrm{K} 2 \cdot \sin ^{2} \varphi \cdot \cos ^{2} \varphi \cdot\left(\left(C_{11}-C_{44}\right) \cdot\left(C_{44}-C_{33}\right)+2 \cdot\left(C_{13}+C_{44}\right)^{2}\right)
\end{array}\right]^{\frac{1}{2}}
\end{gathered}
$$

Der Winkel $\varphi$ charakterisiert hier die Abweichung von der vorgegebenen 3-Richtung, so dass bei entsprechender Ausrichtung der stabförmigen Probekörper $\theta_{1,2}=90^{\circ}-\varphi$ gilt. Für andere Orientierungen des Materials im Koordinatensystem des Modells lassen sich ähnliche Ausdrücke formulieren [17].

\section{Schallabsorption}

In Anlehnung an V. A. Šutilov sei Schallabsorption der Mechanismus, welcher materialspezifisch, unabhängig von der Geometrie der Schallquelle und ihrer Umgebung zu einer Minderung der Energiedichte der Welle und somit der Schallwechseldruckamplitude als Funktion des Ortes führt [19]. Hauptsächliche Ursache für die Absorption ist in den meisten technisch relevanten Medien die innere Reibung, welche sich nach dem Newtonschen Reibungsgesetz näherungsweise durch die der zeitlichen Ableitung der Teilchenverschiebung proportionale viskose Spannung modellieren lässt. Es lässt sich zeigen, dass dieser Ansatz zu komplexen Moduln führt. Die Schallgeschwindigkeiten werden demnach ebenfalls komplex, wobei die Imaginärteile repräsentativ für die exponentiell abklingenden Longitudinal- bzw. Transversalwellen stehen.

Neben der inneren Reibung können Reflexion und Brechung bzw. Beugung und Streuung an Korngrenzen oder Gitterstörungen zu weiteren Frequenzabhängigkeiten der Schallabsorption im Sinne der Definition führen [20]. Diese Effekte sollen vorerst nicht berücksichtigt werden, da sie bei entsprechender Materialauslegung außerhalb des betrachteten Frequenzbereichs stattfinden. Folgende Ausführungen zur frequenzabhängigen Beziehung zwischen Schallgeschwindigkeit und Absorption behalten aber selbst dann ihre Gültigkeit. 


\section{Frequenzabhängige Schallgeschwindigkeit und -absorption}

Leider führt die Annahme komplexer Moduln zwangsläufig zu nicht zeitkausalen Simulationsergebnissen, sei denn die zugrundeliegenden Differentialgleichungen werden zwecks Kausalisierung durch Einbindung eines Faltungskerns modifiziert [21,22]. Solche Implementierungen sind aber längst nicht Bestandteil kommerziell verfügbarer Simulationswerkzeuge. Darin wird meist das sogenannte Rayleigh-Dämpfungsmodell verwendet, welches zumindest für einen eingeschränkten Frequenzbereich und bei genügend geringer Absorption realitätsnahe Ergebnisse liefert. Angesichts seiner hohen Verbreitung soll es auch hier angesetzt werden, ergänzt durch eine frequenzselektive Beschreibung des Zusammenhangs zwischen Schallgeschwindigkeit und -absorption, wie sie nach O'Donnell in den lokalen Kramers-KronigBeziehungen der Akustik zum Ausdruck kommen [23]. Mit dem Lehrschen Dämpfungsmaß K, das bei Annahme des Rayleigh-Dämpfungsmodells als frequenzabhängige Größe durch nur zwei Koeffizienten $\alpha_{\mathrm{M}}$ und $\alpha_{\mathrm{K}}$ ausgedrückt werden kann und der bei einer Frequenz $\omega_{0}$ gemessenen Schallgeschwindigkeit $c_{0}=c\left(\omega_{0}\right)$ gilt also [17]:

$$
\alpha(\omega) \approx \frac{1}{c(\omega)} \cdot \frac{\omega \cdot \mathrm{K}(\omega)}{\sqrt{1-\mathrm{K}(\omega)^{2}}} \wedge c(\omega) \approx c_{0}+\frac{2}{\pi} c_{0}^{2} \cdot \int_{\omega_{0}}^{\omega} \frac{\alpha\left(\omega^{\prime}\right)}{\omega^{\prime 2}} d \omega^{\prime}, \quad \mathrm{K}=\left(\alpha_{\mathrm{M}} \omega^{-1}+\alpha_{\mathrm{K}} \omega\right) / 2
$$

Die nach Einsetzen von $\alpha(\omega)$ verbleibende Volterasche Integralgleichung lässt sich für positive Frequenzen lösen:

$$
\begin{gathered}
c(\omega)=\frac{c_{0}}{\sqrt{\pi}} \cdot \sqrt{\pi-2 \cdot \arctan \left(\alpha_{\mathrm{K}} \frac{B_{0} \sqrt{A}-B \sqrt{A_{0}}}{\alpha_{\mathrm{K}}^{2} \sqrt{A A_{0}}+B B_{0}}\right)+2 j \cdot\left(\ln \frac{C-j \alpha_{\mathrm{M}} \omega^{-2} \sqrt{A}}{C_{0}-j \alpha_{\mathrm{M}} \omega_{0}^{-2} \sqrt{A_{0}}}\right)} \\
\begin{array}{lll}
A=-\omega^{2}(B+C) & A_{0}=-\omega_{0}^{2}\left(B_{0}+C_{0}\right) \\
\text { mit } \quad \begin{array}{l}
B=-2+\alpha_{\mathrm{M}} \alpha_{\mathrm{K}}+\alpha_{\mathrm{K}}^{2} \\
B_{0}=-2+\alpha_{\mathrm{M}} \alpha_{\mathrm{K}}+\alpha_{\mathrm{K}}^{2} \omega_{0}^{2}
\end{array} \\
C=-2+\alpha_{\mathrm{M}} \alpha_{\mathrm{K}}+\alpha_{\mathrm{M}}^{2} \omega^{-2} & C_{0}=-2+\alpha_{\mathrm{M}} \alpha_{\mathrm{K}}+\alpha_{\mathrm{M}}^{2} \omega_{0}^{-2}
\end{array}
\end{gathered}
$$

Damit lässt sich das frequenzabhängige Verhalten sowohl der Schallgeschwindigkeit als auch der Dämpfung durch nur drei Koeffizienten, $\alpha_{\mathrm{M}}, \alpha_{\mathrm{K}}$ und $c_{0}$, beschreiben. Diese Charakteristik gilt es auch bei der Vorwärtsrechnung adäquat zu berücksichtigen.

\section{Modellierung des hohlzylindrischen akustischen Wellenleiters}

Grundlegend für die auch in dieser Arbeit verfolgte inverse Bestimmung der Materialkenngrößen ist ein ganzheitliches Modell der Schallanregung, -ausbreitung und -detektion, welches die schnelle Berechnung der Empfangssignale erlaubt. Die Effizienz dieser Vorwärtsrechnung lässt sich signifikant steigern, wenn möglichst viele Rechenschritte analytisch durchgeführt werden können oder auf einmalig ausgerechnete Teilergebnisse zurückgegriffen werden kann. Diese einmalig berechneten Teilergebnisse sind hier die für verschiedene Querkontraktionszahlen berechneten Dispersionsdiagramme isotroper hohlzylindrischer Probekörper. Variationen der Schallgeschwindigkeiten und Probekörperdurchmesser lassen sich durch Umskalierung berücksichtigen. Die transversale Isotropie wird dann durch einen neuen teilempirischen Ansatz mit einbezogen. Dazu bedarf es zunächst der analytischen Berechnung des zu erwartenden Messeffektes bei ebendieser Anisotropieform.

\section{Messeffekt bei transversaler Isotropie}

Unter Berücksichtigung der angesetzten transversalen Isotropie und der daraus resultierenden richtungsabhängigen Schallgeschwindigkeiten nach GI. 3 und GI. 4 lässt sich dem Strahlenansatz Reynolds' folgend ebenfalls eine Laufzeitdifferenz berechnen [17]:

$$
\Delta t=\frac{D}{c_{3}} \cdot \frac{\sqrt{1-\cos ^{2} \theta_{\mathrm{z}}}}{\cos \theta_{\mathrm{z}}}, \quad \cos \theta_{\mathrm{z}}=\frac{\sqrt{\left(C_{13}^{2}+\left(C_{11}+C_{33}+2 C_{13}\right) C_{44}\right) \cdot C_{11} C_{44}}}{C_{13}^{2}+\left(C_{11}+C_{33}+2 C_{13}\right) C_{44}}, \quad c_{3}=\sqrt{\frac{C_{33}}{\rho}}=\frac{l}{t_{0}}
$$

Allerdings zeigt die Bilanz, dass es bei Kenntnis allein von Laufzeit und Laufzeitdifferenz nicht möglich ist, alle noch unbekannten Moduln $C_{11}, C_{13}$ und $C_{44}$ zu berechnen. Die nur schwer experimentell bestimmbare Größe $C_{44}$ soll Theocaris folgend analytisch genähert werden [7, 17]: 


$$
C_{44}=G_{\mathrm{L}}=\frac{(1+2 \sqrt{2}) \cdot E_{\mathrm{L}}}{2 \cdot\left(1+v_{\mathrm{L}}\right) \cdot\left(2 \sqrt{2}+\left|\frac{E_{\mathrm{T}}+E_{\mathrm{L}} v_{\mathrm{T}}-E_{\mathrm{L}}}{E_{\mathrm{T}} v_{L}}\right|\right)}
$$

Damit ist aber klar, dass es zumindest einer weiteren Messgröße bedarf, zum Beispiel des Amplitudenverhältnisses zwischen erster Signal- und erster Signalfolgegruppe, um eindeutig alle Koeffizienten bestimmen zu können. Allerdings wird das Ampltudenverhältnis auch durch die Dämpfung im Material beeinflusst. Diesen Umstand gilt es zusätzlich zu berücksichtigen.

\section{Simulation der Schallausbreitung im hohlzylindrischen akustischen Wellenleiter}

Nachfolgend sollen exemplarisch einzelne Stationen der Modellierung und Simulation erläutert werden. Den Anfang bildet die Berechnung des Dispersionsdiagramms. Dies erfolgt hier auf Basis der GlobalMatrix-Methode für alle Querkontraktionszahlen und isotrope Medien im gesamten relevanten Frequenzund Wellenzahlbereich, wobei die kleinstmögliche Schallgeschwindigkeit zugrundegelegt wird [24, 25]. Spätere Modifikationen der Probekörperradien sowie Schallgeschwindigkeiten führen dann zu einer Neuskalierung des berechneten Dispersionsdiagramms.

Nach Fourier ist es möglich, jedes beliebige periodische Signal in eine Summe verschieden gewichteter und verschieden frequenter Kosinus- und Sinusschwingungen zu entwickeln. Stellen die freien Schwingungsformen des akustischen Wellenleiters ein Orthogonalsystem dar, ist es möglich jede in seinem Querschnitt vorliegende Schwingungsform auf gleiche Weise in eine gewichtete Summe von Basisfunktionen zu zerlegen (modale Entwicklung). Die angesprochene Skalierung der Dispersionsdiagramme lässt sich auch auf die berechneten modalen Anteilsfaktoren bzw. Gewichte übertragen (Maßstabsänderung der Fouriertransformierten). Bis hierhin müssen die Berechnungen also nur einmalig erfolgen.

Im nächsten Schritt wird die Materialdämpfung hinzugefügt. Bei der modalen Entwicklung zeigt sich, dass bei jeder Frequenz nur wenige, im Wesentlichen sogar nur zwei Wellenleitermoden zur bestmöglichen Rekonstruktion der vorgegebenen mechanischen Spannungen beitragen. Dies zeigt beispielhaft Bild 3, wo die Anteilsfaktoren insgesamt 14 verschiedener Moden über der Frequenz dargestellt sind. Die frequenzabhängige Materialabsorption führt, ausgehend von dieser spektralen Amplitudenverteilung, zu einem ebenfalls frequenzabhängigen Verlauf der modalen Anteilsfaktoren. Die Schwächung jedes einzelnen Frequenzanteils wirkt sich allerdings auch auf seine unmittelbar benachbarten Frequenzanteile aus („Lorentzlinien“). Grundsätzlich ist an dieser Stelle die Vorgabe eines beliebigen Verlaufs der frequenzabhängigen Materialabsorption möglich (Rayleigh, Frequenz-Potenzgesetz, Lorentz-Drude, ...).

Jetzt ist der Moment, wo die zeitkausale Forderung eingebunden werden muss. Mit GI. 6 ist bei Annahme der Rayleigh-Dämpfung auch die frequenzabhängige Schallgeschwindigkeit bekannt, mit der sich der im Dispersionsdiagramm hinterlegte Phasengeschwindigkeitsverlauf für jede Frequenz neu skalieren lässt. Mit der so modifizierten Dispersionsbeziehung und den gedämpften modalen Anteilsfaktoren sind alle

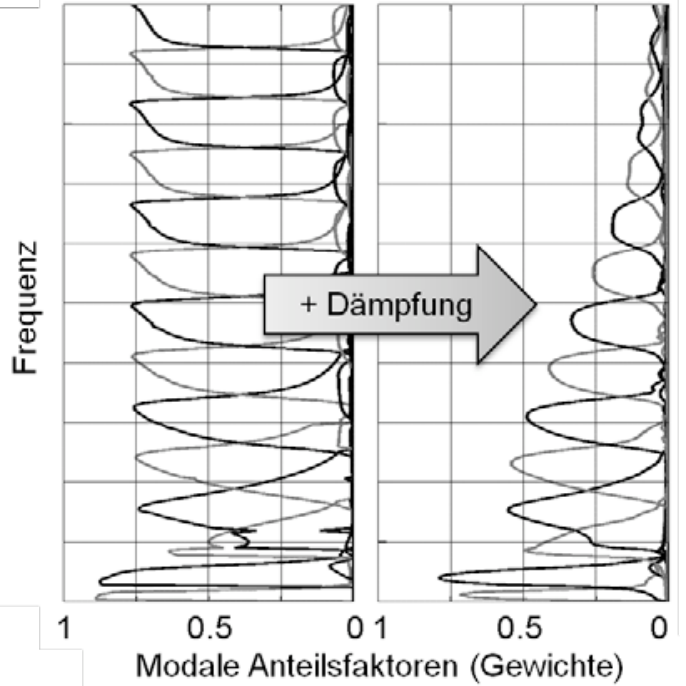

Bild 3: Modale Anteilsfaktoren nach der modalen Entwicklung und mit Vorgabe einer Dämpfung Bausteine zur Beschreibung des akustischen Übertragungskanals vorhanden. Sie können nach Summation über alle betrachteten Moden zu einer einzigen akustischen Übertragungsfunktion $G(\mathrm{j} \omega)$ zusammengefasst werden, wie Bild 4 veranschaulichen soll. Darin sind auch Sender und Empfänger gezeigt, die bei zielgerichteter Konstruktion derselben in guter Näherung als reine Dickenschwinger mit in nur einer Dimension modelliert werden können [17].

Die Berechnung eines Empfangssignals, auch unter zusätzlicher Berücksichtigung der Sender- und Empfängercharakteristik, dauert mit der gezeigten Methode auf einem handelsüblichen Computer nur wenige Sekunden, wobei berechnete und gemessene Signale bei passender Parametrisierung gute Übereinstimmung zeigen. Die Methode ist damit einer herkömmlichen transienten FE-Simulation, selbst unter Annahme der Axialsymmetrie, weit überlegen. 
wenige Sekunden, wobei berechnete und gemessene Signale bei passender Parametrisierung gute Übereinstimmung zeigen. Die Methode ist damit einer herkömmlichen transienten FE-Simulation, selbst unter Annahme der Axialsymmetrie, weit überlegen.

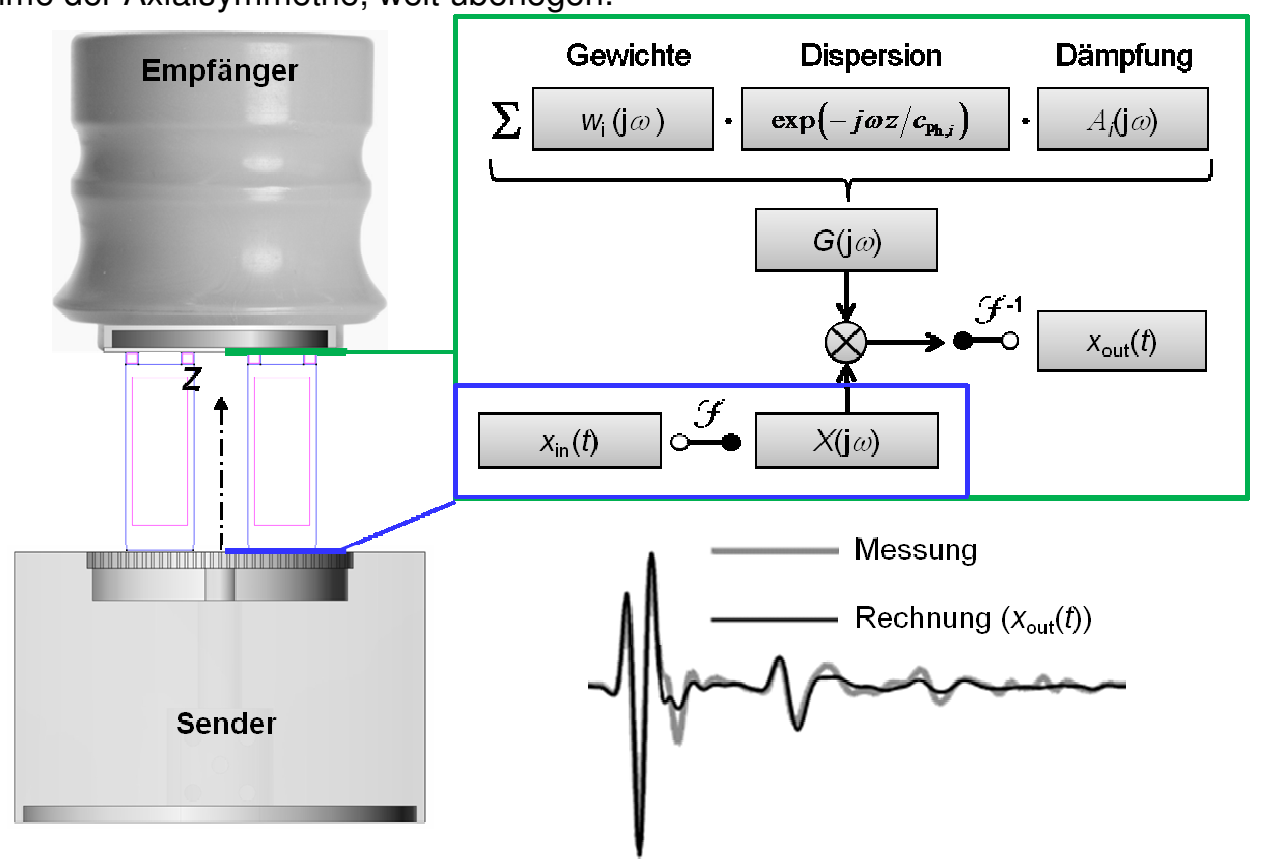

Bild 4: Schematische Darstellung des Versuchsaufbaus sowie der Vorgehensweise zur Berechnung eines Empfangssignals auf Basis der akustischen Übertragungsfunktion und Fourier-Hin- und Rücktransformation.

\section{Annahme schwach anisotroper Verhältnisse}

Es soll hier die schon bekannte Berechnung der Schallausbreitung im isotropen Wellenleiter für die sehr spezielle Anisotropie der transversalen Isotropie genutzt werden. Der Ansatz orientiert sich an der Durchflussmessung mittels Ultraschall, wo in Richtung der Strömung eine um die Strömungsgeschwindigkeit größere Schallgeschwindigkeit angesetzt und daraus resultierend ein Mitnahmeeffekt berechnet wird. Dieser Mitnahmeeffekt sollte auch im transversal isotropen Material zu einem zeitlich verschobenen Empfangssignal führen, nur ohne reale Strömung. Seien $c_{\mathrm{r}}$ und $c_{\mathrm{z}}$ die Schallgeschwindigkeiten im Querschnitt bzw. in Ausbreitungsrichtung, dann wird das Wellenpaket um die Zeit $\Delta t_{1}=l \cdot\left(c_{\mathrm{r}}^{-1}-c_{\mathrm{z}}^{-1}\right)$ mitgenommen. Allerdings hat sich im Vergleich mit FEM-Ergebnissen gezeigt, dass offenbar nicht ausbreitungsfähige Moden bei vorliegender Anisotropie anders abklingen als im isotropen Fall. An dieser Stelle, aber auch im Rahmen der zur Verarbeitung der Materialkonstanten erforderlichen Arithmetik hat sich die Einführung einer empirischen Konstante, eines Anisotropiefaktors $A$, bewährt:

$$
A=\frac{E_{\mathrm{T}} \cdot\left(1-v_{\mathrm{L}}\right)}{E_{\mathrm{L}} \cdot\left(1-v_{\mathrm{T}}\right)}=\frac{C_{11}+C_{12}-C_{13}}{C_{33}}
$$

Je näher dieser Faktor an der Eins liegt, umso mehr ähnelt das Material einem isotropen. Die Quadratwurzel des neuen Anisotropiefaktors gibt in etwa an, wie viel länger oder kürzer ein isotroper Wellenleiter sein müsste, damit die nicht ausbreitungsfähigen Moden auf ein ähnliches Maß abgeklungen sind wie im anisotropen Fall. Die zur Berechnung der Schallausbreitung zu berücksichtigende Länge $\tilde{l}=l / \sqrt{A}$ hat aber auch Folgen für die Gesamtlaufzeit des Signals, welche anschließend um $\Delta t_{2}=(l-\tilde{l}) / c_{\mathrm{r}}$ korrigiert werden muss. Insgesamt bedingt dies im Frequenzbereich die Laufzeitkorrektur $\exp \left(-j \omega t_{\mathrm{k}}\right)$ mit

$$
t_{\mathrm{k}}=\Delta t_{2}-\Delta t_{1}=\frac{l-\tilde{l}}{c_{\mathrm{r}}}-l \cdot\left(\frac{1}{c_{\mathrm{r}}}-\frac{1}{c_{\mathrm{z}}}\right)=l \cdot\left(\frac{1}{c_{\mathrm{z}}}-\frac{1}{\sqrt{A} c_{\mathrm{r}}}\right)
$$

Mit der nach Gl. 7 berechneten Laufzeitdifferenz $\Delta t$ lässt sich ferner eine mittlere Poissonzahl $\bar{v}$ im äquivalenten isotropen Material berechnen: 


\section{Gültigkeitsbereich der teilempirischen Simulationsmethode}

Es hat sich gezeigt, dass die Simulationsergebnisse der neuen teilempirischen Methode für weite Bereiche der Eingangsgrößen $\left(0,15<\bar{v}<0,45\right.$ und $\left.1<c_{\mathrm{z}} / c_{\mathrm{r}}<1,3\right)$ in sehr guter Korrelation $\left(\rho_{x y}>0,95\right)$ zu den weit aufwändiger zu berechnenden FEM-Simulationsergebnissen stehen [17]. Bild 5 zeigt vier ausgewählte durchschnittlich gute Simulationsergebnisse.
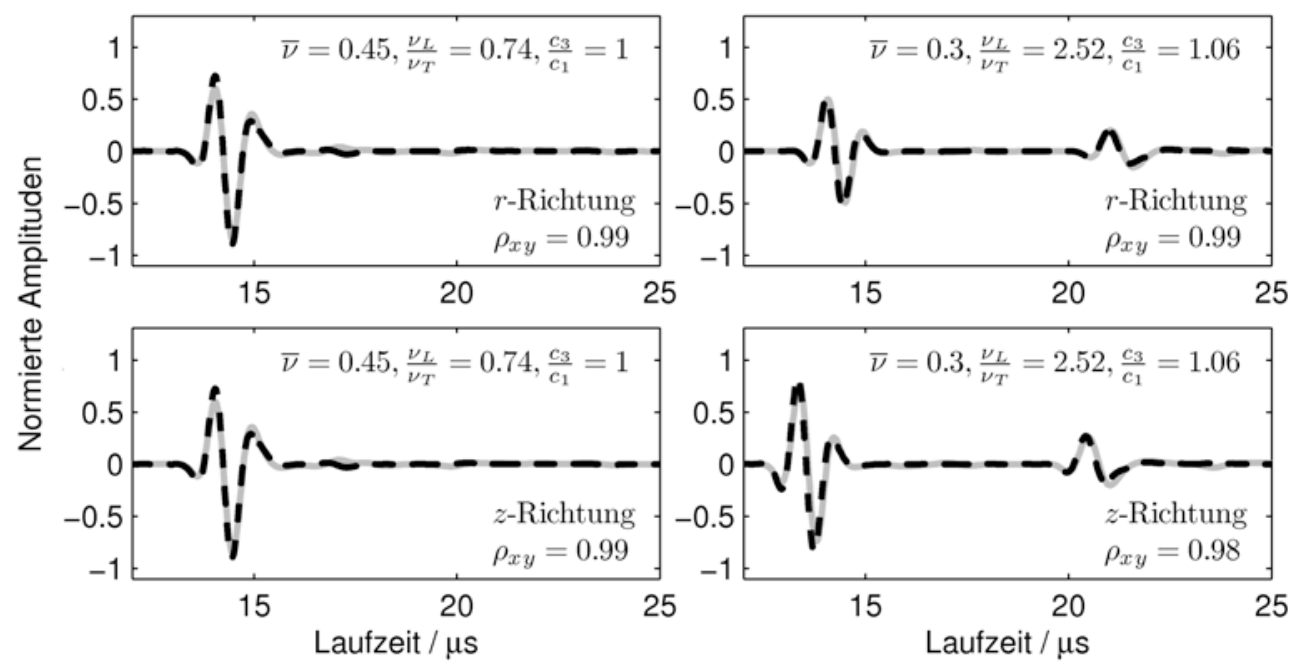

Bild 5: Ausgewählte Simulationsergebnisse für $c_{1}=1350 \mathrm{~ms}^{-1}$ bei $r$ - und $z$ - Ausrichtung der Anisotropie und zwei sehr unterschiedlichen Verhältnissen der Querkontraktionszahlen (grau: FEM, schwarz: Rechnung)

Mit Hilfe des neuen Werkzeugs ist es auch möglich umfangreichere Parameterstudien durchzuführen und sicherzustellen, dass mit einem einheitlichen Probekörper eine möglichst große Bandbreite verschiedener Materialien untersucht werden kann. So ist beispielsweise das Fehlen eines deutlich ausgeprägten Folgeechos, wie links in Bild 5 zu sehen, nicht wünschenswert. Mit einem Innendurchmesser von $6 \mathrm{~mm}$, einem Außendurchmesser von $18 \mathrm{~mm}$ und einer Länge von ca. $20 \mathrm{~mm}$ lässt sich nahezu das gesamte Spektrum verfügbarer Kunststoffe untersuchen. Voraussetzung ist ein hinreichend kurzes Sendesignal von ca. $2 \mu$ s Dauer und ca. $1 \mathrm{MHz}$ Mittenfrequenz.

\section{Messung und Auswertung}

Wie bereits erwähnt, ist die bloße Anwendung von GI. 1 selbst zur Bestimmung der akustischen Kenngrößen eines isotropen und stark dämpfenden Materials nicht hinreichend genau. Deshalb wird hier ein anderer, ein inverser Ansatz gewählt: Ausgehend von einer Anfangskonfiguration der Materialkenngrößen $\left(c_{3}, c_{1}, v_{\mathrm{L}}, v_{\mathrm{T}}, \alpha_{\mathrm{M}}, \alpha_{\mathrm{K}}\right)$ werden diese solange nach einem möglichst effizienten Optimierungsalgorithmus variiert, bis es zu einer bestmöglichen Übereinstimmung der gemessenen und simulierten Signale bzw. ihrer Signalkenngrößen kommt. Nachdem nun die Modellierung und Simulation beschrieben sind, gilt es noch kurz auf den Versuchsaufbau einzugehen und schließlich die Signalkenngrößen bzw. den Bewertungsmaßstab für eine gute Übereinstimmung zu erläutern.

\section{Realisiertes Messsystem}

Der realisierte Versuchsaufbau ist in Anlehnung an den Versuchsaufbau von Reynolds als Transmissionsmessung ausgelegt, wobei die gesamte Anordnung, bestehend aus Ultraschallsender, Probekörper und Ultraschallempfänger in einer Thermokammer steht, mit welcher der Probekörper nahezu gleichmäßig temperiert werden kann. Bild 6 zeigt ein Foto sowie eine Übersicht des Versuchsaufbaus. Die einzige nicht kommerziell verfügbare Komponente ist der Ultraschallsender, welcher eigens für diese Anwendung auf Basis eines piezoelektrischen1-3-Composites erstellt wurde und durch eine spezielle Signalverarbeitung auch bei wechselnden Lastbedingungen nahezu ideal impulsförmige Schallwellen abgeben kann [17]. Bei der Versuchsdurchführung ist darauf zu achten, dass dem Probekörper genügend Zeit gegeben wird, sich der Umgebungstemperatur der Thermokammer anzugleichen. Bei einem Temperatursprung von $5 \mathrm{~K}$ kann dies angesichts sehr geringer Wärmeleitung bis zu 90 Minuten dauern. Dann werden die Transmissionsmessungen durchgeführt, wobei zwischen Burst- und Impulsanregung gewechselt wird. Dadurch können verschiedene Spektralbereiche selektiv angeregt und analysiert werden. 


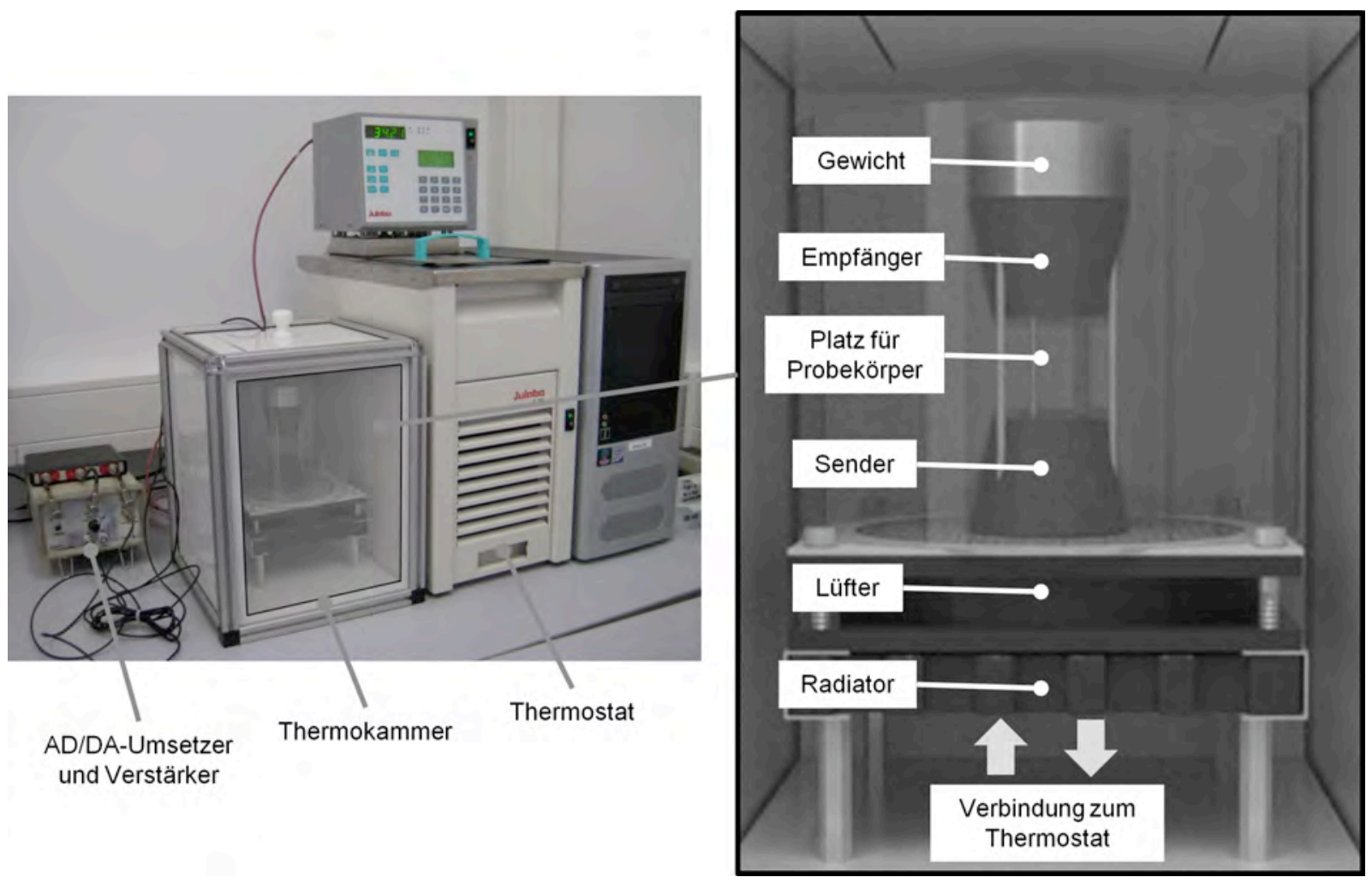

Bild 6: Übersicht und Detailansicht des realisierten Versuchsaufbaus

\section{Bilanz zwischen Freiheitsgraden und Signalkenngrößen}

Angesichts der sechs zu variierenden Freiheitsgrade bedarf es zumindest auch sechs eindeutiger, d.h. weitgehend unabhängiger Merkmale, die sich aus den Empfangssignalen ableiten lassen müssen. Neben der absoluten Laufzeit und der Laufzeitdifferenz zwischen den Signalgruppen sind dies zum Beispiel das Amplitudenverhältnis zwischen erster Signalgruppe und erster Signalfolgegruppe, die Lage des Frequenzschwerpunktes $f_{\mathrm{S}}$ sowie die Bandbreite $2 \sigma_{P}$ am Ende des Wellenleiters und die Periodizität des Spektrums in Form seiner lokalen Extrema $f_{\mathrm{c}, m}$ (Modeordnung $m$ ). Um diese sehr verschiedenen Kriterien mit ähnlicher Bedeutsamkeit in einer zu minimierenden Zielfunktion $\varepsilon\left(\begin{array}{l}1 \\ x\end{array}\right)$ zusammenfassen zu können, bedarf es einer Skalierung

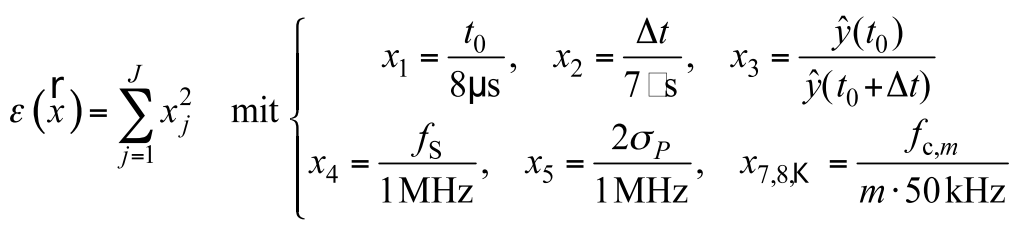

\section{Ergebnisse}

Die Minimierung der in Gl. 12 genannten Zielfunktion führt zu einem Modellparametersatz, der sich nach den oben genannten Formeln für jede untersuchte Temperatur in die frequenz- und richtungsabhängigen Größen umrechnen lässt. Die Ergebnisse dieser Umrechnung sollen nachfolgend am Beispiel von Polypropylen (PP) dargestellt werden. In Bild 7 sind die Ergebnisse für $20^{\circ} \mathrm{C}$ jeweils blau dargestellt, die für $50^{\circ} \mathrm{C}$ in rot, Graphen für die Transversalwelle $c_{\mathrm{T}}$ gestrichelt und für die Longitudinalwelle $c_{\mathrm{L}}$ mit durchgezogenen Linien.

Hier ist zu erkennen, dass die Longitudinalwellengeschwindigkeit im Temperaturbereich von $20^{\circ} \mathrm{C}$ bis $50^{\circ} \mathrm{C}$ um ca. $15 \%$, die Transversalwellengeschwindigkeit bereits um ca. $25 \%$ sinkt. Noch größer sind die temperaturbedingten Änderungen der Schallabsorption (ausgedrückt durch den Schwächungskoeffizienten), was sich in ebenfalls großer Frequenzabhängigkeit wiederfindet. 

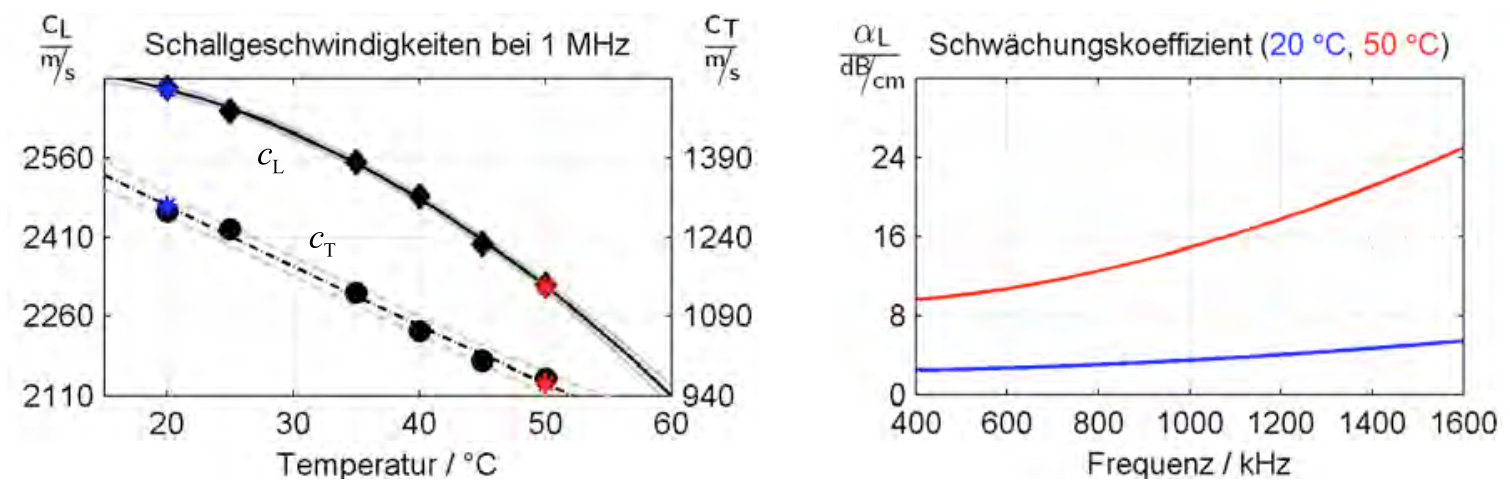

Bild 7: Schallgeschwindigkeiten und Schwächungskoeffizient von Polypropylen (PP) als Funktion der Temperatur und Frequenz

PP hat sich bei allen Temperaturen als nahezu isotrop herausgestellt, was die in Bild 8 gezeigte Slowness-Kurve (Kehrwert der Schallgeschwindigkeit, berechnet nach GI. 3 und GI. 4) anschaulich zeigt. Trotz dieser Eigenschaft und des damit verbundenen vergleichsweise einfachen Modells stellt sich heraus, dass sich die Signale bei Annahme des Rayleigh-Dämpfungsmodells nicht in der gewohnt guten Qualität berechnen lassen. Entweder lässt sich die erste Signalgruppe (korrespondierend mit den Longitudinalwellen) oder aber die Folgegruppen (korrespondierend mit den Transversalwellen) besonders gut abbilden. Die gleich gute Abbildung beider Gruppen gelang bislang nicht. Den besten Kompromiss zeigt das nachfolgend dargestellte berechnete Signal.
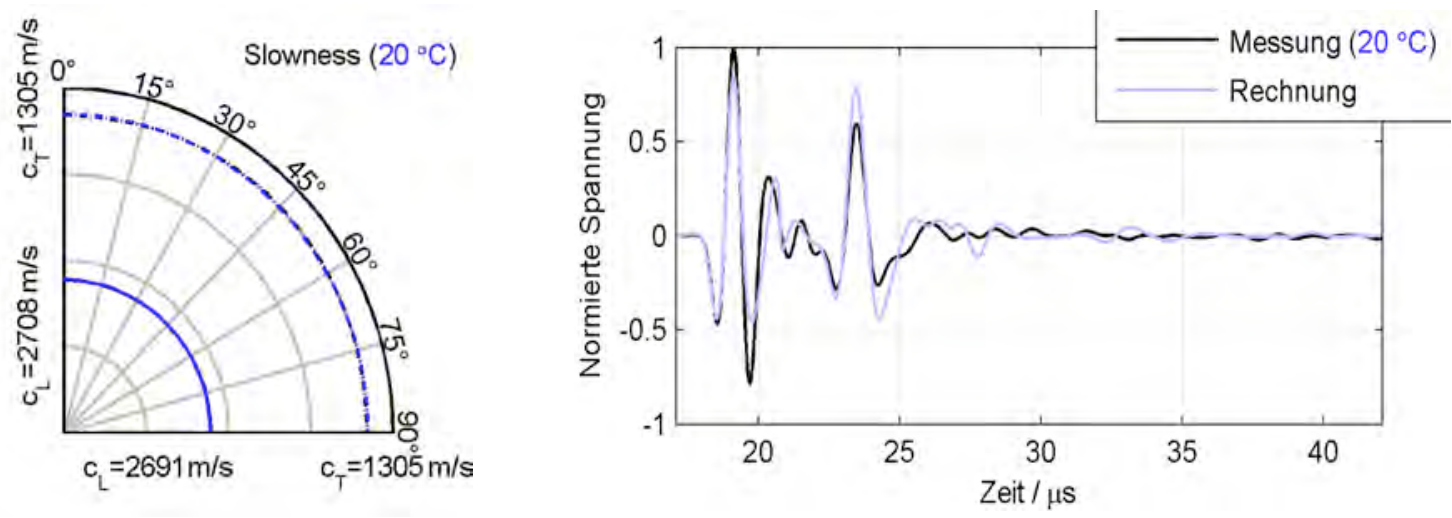

Bild 8: Slowness-Kurve von Polypropylen und Vergleich zwischen simuliertem und gemessenem Signal

Polypropylen ist damit ein gutes Beispiel dafür, dass trotz Maßnahmen zur Kausalisierung und Berücksichtigung frequenzabhängiger Dämpfung das Rayleighmodell nicht gut genug ist, die Ausbreitung eines breitbandigen Signals in einem stark absorbierenden Mediums zu modellieren.

\section{Zusammenfassung}

Es wurde ein inverses Verfahren vorgestellt, mit dem es möglich ist, anhand einer einzigen Transmissionsmessung durch einen akustischen Wellenleiter alle Daten zu bestimmen, die für die Simulation von Schallausbreitungsphänomenen in stark absorbierenden und schwach anisotropen Materialien erforderlich sind. Dazu wurden zunächst die erforderlichen Freiheitsgrade zur Beschreibung eines transversal isotropen Materials eingeführt und Möglichkeiten zur zeitkausalen Modellierung und schnellen Simulation der Schallausbreitung im akustischen Wellenleiter aufgezeigt. Grundlegender Messeffekt ist die mehrmalige Modekonversion an den Mantelflächen eines Hohlzylinders, wonach ein Empfangssignal verschiedene Signalgruppen mit definierten Laufzeitdifferenzen aufweist. Diese konnten auch bei der angesetzten Materialsymmetrie analytisch berechnet werden, was grundlegend für die effiziente teilempirische Vorwärtsrechnung des inversen Algorithmus ist. Insgesamt konnten sechs weitgehend unabhängige Signalkenngrößen identifiziert werden, um die sechs verleibenden Material-Freiheitsgrade des angesetzten Modells zu charakterisieren. Am Beispiel von Polypropylen wurden akustische Kenngrößen dargestellt, die sich aus den invers genäherten Materialkenngrößen direkt ableiten lassen. Bei der Vorwärtsrechnung mit 
diesen Größen wurden aber auch Schwächen des angesetzten Rayleigh-Dämpfungsmodells offenbar, die im Kontext dieser Arbeit nur durch Ansatz realitätsnäherer Dämpfungsmodelle zu überwinden sind.

\section{Referenzen}

[1] V. M. Ushakov: Principles of the Development of Piezoelectric Transducers for Ultrasonic Testing of Articles with Flat and Curvilinear Surfaces, In: Rus. J. Nondestructive Testing, Vol. 41, Nr. 6, pp. 355-361, 2005

[2] F. Wolf, T. LAHMER, L. BAhr, A. HAuck, A. Sutor, R. LeRCH, M. KaltenBacher: Finite Element Modeling of Ultrasonic Transducer by Utilizing an Inverse Scheme for the Determination of its Material Parameters, In: IEEE Int. Ultras. Symposium Proc., pp. 784-787, 2008

[3] B. Henning, J. Rautenberg, C. Unverzagt, A. Schröder, S. Olfert: Computer-assisted design of transducers for ultrasonic sensor systems, In: Meas. Sci. Technol., Vol. 20 (124012), Issue 12, 2009

[4] M. KALTENBACHER: Numerical Simulation of Mechatronic Sensors and Actuators, Springer-Verlag, Berlin 2004, ISBN: 978-3-540-20458-9

[5] S. S. Shashidhara Reddy, K. Balasubramaniam, C. V. KRIShnamurthy, M. Shankar: Ultrasonic goniometry immersion techniques for the measurement of elastic moduli, In: Composite Structures, Vol. 67, pp. 3-17, 2005

[6] J. VishnUVARDHAN, C. V. KRISHNAMURTHY, K. BALASUBRAMANIAM: Genetic algorithm reconstruction of orthotropic composite plate elastic constants from a single non-symmetric plane ultrasonic velocity data, In: Composites, Vol. 38, pp. 216-227, 2007

[7] P. S. THEOCARIS: Sorting out the elastic anisotropy of transversely isotropic materials, In: Acta Mechanica 143, Springer-Verlag, pp. 129-140, 2000

[8] M. CAstaings, B. Hosten, T. KUndu: Inversion of ultrasonic, plane-wave transmission data in composite plates to infer viscoelastic material properties, In: NDT\&E Int., Vol. 33, pp. 377-392, 2000

[9] A. G. EVERY, W. SACHSE: Determination of the elastic constants of anisotropic solids from acoustic-wave group-velocity measurements, In: Physical Review B, Vol. 42, Nr. 13, 1990

[10] B. Castagnede, K. Y. KIM, W. Sachse, M. O. Thompson: Determination of the elastic constants of anisotropic materials using laser-generated ultrasonic signals, In: J. Appl. Phys., Vol. 70, Nr. 1, 1991

[11] J. VISHNUVARDHAN, C. V. KRISHNAMURTHY, K. BALASUBRAMANIAM: Genetic algorithm based reconstruction of the elastic moduli of orthotropic plates using an ultrasonic guided wave single-transmitter-multiple-receiver SHM array, In: Smart Mater. Struct., Vol. 16, pp. 1639-1650, 2007

[12] W. P. Rogers: Elastic Property Measurement Using Rayleigh-Lamb Waves, In: Res. Nondestr. Eval., Vol. 6, pp. 185-208, 1995

[13] K. HELLER, L. J. JACOBS, J. QU: Characterization of adhesive bond properties using Lamb waves, In: NDT\&E International, Vol. 33, pp. 555-563, 2000

[14] M. SALE, P. RIzZO, A. MARZANI: Semi-analytical formulation for the guided waves-based Reconstruction of elastic moduli, In: Mech. Systems and Signal Processing, Vol. 25, pp. 2241-2256, 2011

[15] M. B. REYNolds: The Determination of the Elastic Constants of Metals by the Ultrasonic Pulse Technique, In: Trans. Am. Soc. for Metals, 45, pp. 839-861, 1953

[16] K. F. GrafF: Wave motion in elastic solids, Dover Publications, Mineola 1991, ISBN 0-486-66745-6

[17] J. RAUTENBERG: Ein Wellenleiter basiertes Verfahren zur Bestimmung von Materialdaten für die realitätsnahe Simulation von Schallausbreitungsphänomenen am Beispiel stark absorbierender Kunststoffe, Dissertation, Paderborn 2011 (unveröffentlicht, laufendes Promotionsverfahren)

[18] S. S. S. REDDY, K. BALASUBRAMANIAM, C.V. KRISHNAMURTHY, M. SHANKAR: UItrasonic goniometry immersion techniques for the measurement of elastic moduli, In: Comp. Structures, Vol. 67, Elsevier, pp. 3-17, 2005 V. A. ŠutILoV: Physik des Ultraschalls, Springer-Verlag, Wien 1984, ISBN: 3-211-81798-0 H. KutTRUfF: Akustik - Eine Einführung, S. Hirzel Verlag, Stuttgart, Leipzig 2004, ISBN 3-7776-1244-8

L. BAHR, M. KALTENBACHER, R. LeRCH: Determination of damping and dispersion parameters with a combined measurement-simulation technique, In: IEEE Ultrasonics Symposium 2005, Proceedings, pp. 1687-1690

K. R. WAters, J. Mobley, J. G. MilleR: Causality-imposed (Kramers-Kronig) Relationships Between Attenuation and Dispersion, In: IEEE Transactions on Ultrasonics, Ferroelectrics and Frequency Control, Vol. 52 (5), 2005, pp. 822-833

[23] M. O'DONNELL, E. T. JAYNES, J. G. MILLER: Kramers-Kronig relationship between ultrasonic attenuation and phase velocity, In: Journal of the Acoustical Society of America, Vol. 69 (3), 1981, pp. 696-701 F. BAUSE, J. RAUtenBeRg, B. HenNing: An improved mode-tracing algorithm to compute dispersion curves of acoustic waveguides, In: IEEE Ultrasonics, 11.-14-10.2010, San Diego, Proceedings, pp. 719-722

[25] M. J. S. LowE: Matrix Techniques for Modeling Ultrasound Waves in Multilayered Media, In: IEEE Trans. on Ultras. Ferroel. and Frequency Control, Vol. 42, No. 4, 1995 